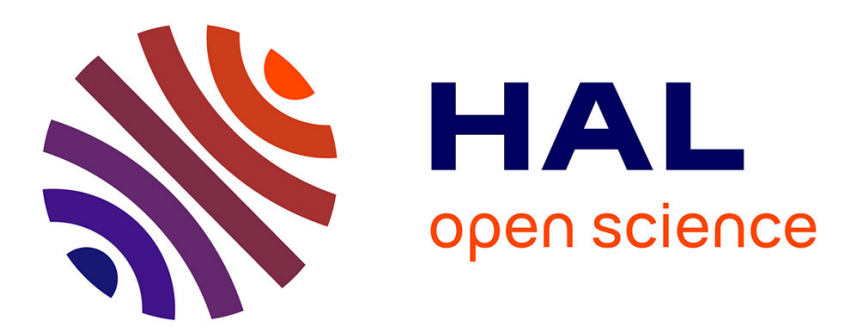

\title{
Optical Bloch modeling of femtosecond-laser-induced electron dynamics in dielectrics
}

\author{
E. Smetanina, P. González de Alaiza Martínez, I. Thiele, B. Chimier, A \\ Bourgeade, G. Duchateau
}

\section{- To cite this version:}

E. Smetanina, P. González de Alaiza Martínez, I. Thiele, B. Chimier, A Bourgeade, et al.. Optical Bloch modeling of femtosecond-laser-induced electron dynamics in dielectrics. Physical Review E , 2020, 101, 10.1103/physreve.101.063206 . hal-02992105

\section{HAL Id: hal-02992105 \\ https://hal.science/hal-02992105}

Submitted on 6 Nov 2020

HAL is a multi-disciplinary open access archive for the deposit and dissemination of scientific research documents, whether they are published or not. The documents may come from teaching and research institutions in France or abroad, or from public or private research centers.
L'archive ouverte pluridisciplinaire HAL, est destinée au dépôt et à la diffusion de documents scientifiques de niveau recherche, publiés ou non, émanant des établissements d'enseignement et de recherche français ou étrangers, des laboratoires publics ou privés. 


\title{
Optical Bloch modeling of femtosecond-laser-induced electron dynamics in dielectrics
}

\author{
E. Smetanina, ${ }^{1,2,3}$ P. González de Alaiza Martínez, ${ }^{1}$ I. Thiele, ${ }^{4}$ B. Chimier, ${ }^{1}$ A. Bourgeade, ${ }^{1}$ and G. Duchateau $\odot^{1, *}$ \\ ${ }^{1}$ Université de Bordeaux, CNRS, CEA, Centre Lasers Intenses et Applications, UMR5107, 33405 Talence, France \\ ${ }^{2}$ Department of Physics, University of Gothenburg, SE-412 96 Göteborg, Sweden \\ ${ }^{3}$ Faculty of Physics, M.V. Lomonosov Moscow State University, 119313 Moscow, Russia \\ ${ }^{4}$ Department of Physics, Chalmers University of Technology, SE-412 96 Göteborg, Sweden
}

(Received 29 April 2020; accepted 12 June 2020; published 29 June 2020)

\begin{abstract}
A model based on optical Bloch equations is developed to describe the interaction of femtosecond laser pulses with dielectric solids, accounting for optical-cycle-resolved electron dynamics. It includes the main physical processes at play: photoionization, impact ionization, direct and collisional laser heating, and recombination. By using an electron band structure, this approach also accounts for material optical properties as nonlinear polarization response. Various studies are performed, shedding light on the contribution of various processes to the full electron dynamics depending on laser intensity and wavelength. In particular, the standard influence of the impact ionization process is retrieved.
\end{abstract}

DOI: 10.1103/PhysRevE.101.063206

\section{INTRODUCTION}

Modern laser technologies provide high-intensity femtosecond laser pulses which open new doors for studies of laser-matter interaction processes. In case of semiconductors or dielectric material targets, such laser pulses can be used to drive the electronic populations in excited states, then allowing various applications including high-order harmonic generation [1,2], THz radiation [3-5], material ablation, and surface texturing [6-8]. Another promising application concerns an initially insulating dielectric material which can be reversibly driven to a conducting state within a femtosecond temporal resolution, paving the way to $\mathrm{PHz}$ electronics [9]. A decrease in the pulse duration, together with beam shaping techniques including utilization of broad spectrum or spatiotemporal chirp, is expected to increase the efficiency of such applications [10-14].

To support such developments, widely used modeling approaches, describing the laser-induced electron dynamics by femtosecond pulses in dielectric materials, currently rely on optical cycle-averaged electron transition rates [15-18]. These approaches are not well adapted to the present context. A model accounting for the optical-cycle-resolved electron dynamics is needed. Such a time-dependent model has to account for the photoionization and the electron dynamics in the conduction band where electrons may further absorb photons and undergo collisions [19]. The latter leads to energy relaxation of excited electrons toward the lattice, inducing an energy deposition into the material and subsequent modifications [20-22]. This model should also be suitable for its implementation into a Maxwell solver [19,23-26] to describe the coupled laser propagation and electron dynamics that generally take place for such physical systems [10,19,26-29].

*guillaume.duchateau@u-bordeaux.fr
A candidate fulfilling previous requirements relies on the numerical resolution of optical Bloch equations (OBE) where the time-dependent laser electric field is the input parameter $[23,28]$. Nevertheless, the impact ionization process was not tackled, neither an in-depth treatment of electron collisions whereas their influence is crucial since they lead to decoherence effects and to the laser energy deposition into the material. On the other hand, kinetic-type descriptions have been developed, accounting for all main collisional processes $[30,31]$. But they generally do not account for the band structure beyond a single parabolic band and are currently computationally too expensive for their coupling to a Maxwell solver. First-principle approaches as time-dependent density functional theory fully describe the band structure, as well as the time-dependent interaction $[32,33]$. However collisions are not properly described $[34,35]$. To our knowledge, there is no model including the following required features to support above-mentioned developments: time-dependent (cycleresolved) laser electric field as input of the model, description of the band structure, description of collisions, photoionization, electron heating in the conduction band, description of impact ionization, and electron recombination.

The goal of the present work is to propose such a model which ultimately aims at being implementing into a Maxwell solver, demonstrate its reliability, and provide a first study within this framework, exhibiting in particular the role of the impact ionization which contribution on the electron dynamics within an optical-cycle-resolved approach was never tackled to our knowledge. A study describing all interaction processes, including laser propagation effects and macroscopic material response as hydrodynamics and heat diffusion, is out of the scope of the present work, some results having been reported in Refs. [21,36-41]. The proposed model describing the full time-dependent electron dynamics, and including various material characteristics, is based on OBE. It consists in solving the Liouville-von Neumann equation for the density matrix (Sec. II). A band structure is introduced through a set 
of energy levels. By coupling appropriately those levels, photoionization, impact ionization, transitions in the conduction band (laser heating), and electron recombination are taken into consideration. The numerical solution of these equations for the density matrix provides the time-dependent electron population for each energy level, allowing one to evaluate the ionized electron density and the free electron energy density, as well as both linear and nonlinear polarization responses. In order to demonstrate the ability of the developed OBE model to account for main features of the femtosecond laser-induced electron dynamics in dielectric solids, the widely studied fused silica have been chosen with both centrosymmetric and noncentrosymmetric configurations. Since the presented model is general, material parameters are provided independently of the model description (Sec. III). Within this framework, the pure photoionization, the polarization, and the full electron dynamics are studied in Sec. IV. The reliability of the OBE approach is demonstrated by the above-mentioned cases of interest where standard trends are retrieved. Conclusions and outlooks are drawn in Sec. V. For the reader convenience, details of the numerical scheme used to solve OBE is provided in Appendix.

\section{OBE MODEL DESCRIPTION}

The admitted picture of the electron dynamics induced by femtosecond laser pulses in dielectric materials is as follows. The initial photoionization process corresponds to valence electrons which are promoted to the bottom of the conduction band through multiphoton absorption or tunneling. The produced conduction electrons may further absorb photons through both direct transitions to excited states with higher energy or phonon-, ion-, or electron-assisted collisions [16,42]. Electron collisions with other particles, without involving photon absorption, may also take place. That first leads to a change in the electron energy distribution. Second, diffusion processes induce a decoherence between the electron and the laser electric field due to momentum exchange, which is hereafter referred to as coherence loss [43]. Finally, conduction electrons may recombine to the valence band.

The OBE are constructed from the Liouville-von Neumann equation which describes the time evolution of a quantum system using the density matrix formalism. To include impact ionization and various relaxation processes taking place in laser irradiated dielectric materials, the equation driving the evolution of the density matrix, $\hat{\rho}$, reads $[23,26]$ :

$$
\partial_{t} \hat{\rho}=\hat{\mathcal{L}}(\hat{\rho})+\hat{\mathcal{G}}_{\mathrm{r}}(\hat{\rho})+\hat{\mathcal{G}}_{\text {imp }}(\hat{\rho}),
$$

where $\hat{\mathcal{L}}(\hat{\rho})$ is the so-called Liouville-von Neumann operator accounting for direct laser-induced transitions. The operators $\hat{\mathcal{G}}_{\mathrm{r}}(\hat{\rho})$ and $\hat{\mathcal{G}}_{\text {imp }}(\hat{\rho})$ introduce the electron relaxation (i.e., recombination and coherence loss) and impact ionization, respectively. The numerical scheme developed to solve the present OBE model given by Eq. (1) is presented in Appendix. It conserves the matrix trace, i.e., the total number of electrons. It has been checked in numerical simulations.

Note that in Eq. (1), the term including $\nabla_{\mathbf{k}}$, where $\mathbf{k}$ is the wave vector, has been removed in order to develop a computationally efficient approach well suited for its coupling with a Maxwell solver. The present model thus does not include the dispersion relation of bands. For the ionization process, it implies that resonant conditions (band gap is a multiple of the photon energy) should be used for which main electron transitions take place in the center of the Brillouin zone. Regarding the electron dynamics in the conduction band, the $\nabla_{\mathbf{k}}$ term accounts for intraband transitions and can play a role in general $[44,45]$. However interband transitions is expected to be the main process leading to high-energy electrons able to induce impact ionization, so that only these transitions are considered within the present goal to highlighting the ability of the present $\mathrm{OBE}$ to correctly describe the impact ionization.

\section{A. Liouville-von Neumann operator}

The Liouville-von Neumann operator $\hat{\mathcal{L}}(\hat{\rho})$ is given by [23]:

$$
\hat{\mathcal{L}}(\hat{\rho}):=-\frac{i}{\hbar}[\hat{H}, \hat{\rho}]=-\frac{i}{\hbar}(\hat{H} \hat{\rho}-\hat{\rho} \hat{H}),
$$

where $\hat{H}(t)$ is the electron Hamiltonian which reads:

$$
\hat{H}(t)=\hat{H}_{0}+\hat{V}(t) .
$$

The unperturbed Hamiltonian $\hat{H}_{0}$ is modeled as a diagonal matrix which elements are the energy levels of the electronic system. We consider a finite number $N$ of allowed energy levels in the conduction band. The corresponding index for each state is $j$ with $0<j \leqslant N$. Depending on the material properties (defined in Sec. III), the valence band contains one single level with the index $j=0$, or two energy levels (with almost the same energy) with indexes $j=-1$ and $j=0$. For example, in case of one valence band state, the unperturbed Hamiltonian reads:

$$
\hat{H}_{0}=\left[\begin{array}{cccc}
E_{0} & 0 & 0 & 0 \\
0 & E_{1} & 0 & 0 \\
0 & 0 & \ddots & 0 \\
E_{0} & 0 & 0 & E_{N}
\end{array}\right] .
$$

The levels in the conduction band have been chosen assuming two adjacent states are resonantly bridged by one photon, i.e., assuming the density of states is large enough to always fulfill resonant conditions [16]. These energy levels thus read:

$$
E_{j}=E_{g}+(j-1) \hbar \omega_{0},
$$

where $\hbar \omega_{0}$ is the photon energy of the incident light and $E_{g}$ is the band-gap energy of the considered material.

The energy of the highest level, $j=N$, is chosen such that the impact ionization process fulfills the energy conservation [16]. For the sake of simplicity, we assume that the electron mass in both valence and conduction bands is the free electron mass, and the contribution of the ponderomotive energy is neglected, leading to:

$$
E_{N}-E_{1} \simeq \frac{3}{2} E_{g} .
$$

The number of levels in the conduction band then is

$$
N=1+\left\lfloor\frac{3 / 2 E_{g}}{\hbar \omega_{0}}\right\rfloor,
$$

where $\lfloor x\rfloor$ is the floor function (maximum integer that is less or equal to $x$ ). 
The interaction Hamiltonian $\hat{V}$ in Eq. (3) is calculated with the electric dipolar approximation in the length gauge as follows:

$$
\hat{V}(t)=-e F(t) \hat{\mu},
$$

where $e$ is the elementary electric charge, $F(t)$ is the laser electric field, and $\hat{\mu}$ is the dipolar transition matrix (which is Hermitian). Its elements reads $\mu_{i j}=\left\langle\varphi_{i}|x| \varphi_{j}\right\rangle$, where $\left|\varphi_{i}\right\rangle$ is the quantum $i$ state, and $x$ is the position operator. Within the present approach, since the explicit expression of wave functions is unknown, each dipolar matrix element is a free parameter to be set (see Sec. III). Note that depending on the state, the wave function may exhibits a well-defined parity $[\varphi(-x)=\varphi(x)$ or $\varphi(-x)=-\varphi(x)]$ or not. Then $\mu_{i i}=0$ or $\mu_{i i} \neq 0$, respectively.

\section{B. Relaxation operator}

Two relaxation processes are considered in Eq. (1), namely recombination $\left(\hat{\mathcal{G}}_{\text {rec }}\right)$ and relaxation of coherence $\left(\hat{\mathcal{G}}_{\text {coh }}\right)$ :

$$
\hat{\mathcal{G}}_{\mathrm{r}}(\hat{\rho})=\hat{\mathcal{G}}_{\mathrm{rec}}(\hat{\rho})+\hat{\mathcal{G}}_{\mathrm{coh}}(\hat{\rho}) .
$$

The electron recombination process is introduced as a decay of conduction electrons to the valence band on the timescale of $\tau_{\text {rec }}$. The equations describing the recombination process in case of one valence band level are as follows:

$$
\hat{\mathcal{G}}_{\mathrm{rec}}(\hat{\rho}) \equiv\left\{\begin{array}{l}
\partial_{t} \rho_{j, j}=-\rho_{j, j} / \tau_{\mathrm{rec}}, \quad \text { for } 1 \leqslant j \leqslant N, \\
\partial_{t} \rho_{0,0}=\sum_{j=1}^{N} \rho_{j, j} / \tau_{\mathrm{rec}} .
\end{array}\right.
$$

If there are two valence band levels with similar energy, then conduction electrons recombine indifferently to any of these two levels.

The coherence loss due to electron collisions is modeled by an exponential decay of the off-diagonal density matrix elements [43]:

$$
\hat{\mathcal{G}}_{\mathrm{coh}}(\hat{\rho}) \equiv\left\{\partial_{t} \rho_{j, k}=-\rho_{j, k} / \tau_{\mathrm{coh}}, \quad \text { for } j \neq k,\right.
$$

where $\tau_{\text {coh }}$ is the coherence-loss characteristic timescale.

\section{Impact ionization operator}

The impact ionization operator in Eq. (1), $\hat{\mathcal{G}}_{\text {imp }}$, describes how the collision of an electron in the highest conduction band level with a valence electron results in both electrons into the lowest conduction band level, through energy transfer. The corresponding equations in case of one valence band level read:

$$
\hat{\mathcal{G}}_{\text {imp }}(\hat{\rho}) \equiv\left\{\begin{array}{l}
\partial_{t} \rho_{N, N}=-\rho_{N, N} / \tau_{\text {imp }} \\
\partial_{t} \rho_{1,1}=2 \rho_{N, N} / \tau_{\text {imp }} \\
\partial_{t} \rho_{0,0}=-\rho_{N, N} / \tau_{\text {imp }}
\end{array}\right.
$$

where $\tau_{\mathrm{imp}}$ is the characteristic impact collision timescale. In case of two valence band levels, each state contributes equivalently to the impact ionization process (with the previous collision timescale).

\section{MATERIAL DESCRIPTION}

As a wide application of the present model, we consider two dielectrics with characteristic parameters similar to silica: $E_{g}=9 \mathrm{eV}$, an initial density of neutral atoms $N_{0}=2.2 \times$ $10^{22} \mathrm{~cm}^{-3}$ [46], $\tau_{\text {rec }} \sim 150$ fs $[47,48], \tau_{\text {imp }} \sim 1$ fs [45], and $\tau_{\text {coh }} \sim 1$ fs [49]. To further illustrate the model ability to account for material properties, the first material is centrosymmetric (like fused silica) and the other is noncentrosymmetric (like quartz).

For the centrosymmetric material, two valence band levels are considered, namely $j=-1$ and $j=0$, separated by an energy $\Delta E_{-1,0} \ll E_{g}$. In practice we have chosen $\Delta E_{-1,0}=$ $0.01 \mathrm{eV}$ which is much smaller than the photon energy and the band gap. The wave functions associated to a given valence energy level are assumed to have a well-defined parity (valence electrons are considered as described by a bound atomic state with a given orbital), hence $\mu_{j, j}=0$. Both the elements $\mu_{-1,0}=2 \AA$ and $\mu_{0,1}=0.5 \AA$ have been set in order to retrieve the Keldysh ionization rate at $\hbar \omega=1.5 \mathrm{eV}$ [15]. Since each conduction level does not have a well-defined parity (assuming conduction electrons may be described by plane waves), each valence level is bridged to any conduction state. In practice we set:

$$
\mu_{l, j>1}=\mu_{j>1, l}=\mu_{0,1} \frac{E_{1}-E_{l}}{E_{j}-E_{l}},
$$

where $l=0,-1$. The transitions in the conduction band, accounting for the laser electron heating, are allowed between all states. However, the larger the energy difference, the lower the transition probability. To account for this fact, by considering conduction electrons as plane waves, the conductionconduction matrix elements may be approximated by:

$$
\mu_{j, k}=\mu_{k, j}=\mu_{\mathrm{CB}} \frac{1}{\left|E_{j}-E_{k}\right|},
$$

where $j>0, k>0$, and $j \neq k . \mu_{\mathrm{CB}}$ is set to $0.45 \mathrm{eV} \AA$ in order to account for standard one-photon absorption cross section in the conduction band [16].

For the noncentrosymmetric material, only one valence level is considered with a wave function that does not have a well-defined parity, leading to $\mu_{0,0} \neq 0$. As previously, it is set to $2 \AA$ to retrieve the Keldysh ionization rate. The transitions from this valence state to conduction levels is described as previously, i.e., matrix elements are given by Eq. (13) with $l=$ 0 . Regarding transitions in the conduction band, the model is similar to the centrosymmetric material.

Finally, it is worth noting that these values of the transition matrix element are reasonable in the sense that they are in the Angström range corresponding to the expected value of the quantum position operator in solids. They allow one to catch the main physical mechanisms and account for the main trends for the laser-induced electron dynamics in dielectric materials. Slight variations of the above parameters do not change the forthcoming conclusions.

\section{RESULTS AND DISCUSSION}

Based on the previous descriptions, this section is devoted to the numerical predictions of the OBE and their analysis. In 
order to highlight the various physical processes at play and the capability of the present approach, various configurations are considered. First, in Sec. IV A, only the photoionization mechanism is allowed by using two or three energy levels to account for centrosymmetric or noncentrosymmetric materials. Such a configuration allows one also to evaluate the material polarization response as presented in Sec. IV B. Finally the full electron dynamics, where the conduction band is described by a set of states, is addressed in Sec. IVC. To analyze the results, the physical quantities of interest are the produced electron density in the conduction band, and its energy density. The conduction electron density is estimated as the probability of finding an electron in the conduction band weighted by the initial density of valence electrons, $N_{0}$ :

$$
N_{e}(t)=N_{0} \sum_{j>0}^{N} \rho_{j, j}(t) .
$$

The ionization degree is defined as $Z=\frac{N_{e}\left(\tau_{0}\right)}{N_{0}}$, where $\tau_{0}$ is the instant when the laser pulse ceases. The initial condition for noncentrosymmetric materials (the valence band only consists of one energy level $j=0)$ is $\rho_{0,0}(t=0)=1$. In the case of a centrosymmetric material, the two energy levels $j=-1$ and $j=0$ are initially filled as $\rho_{-1,-1}(t=0)=\rho_{0,0}$ $(t=0)=1 / 2$.

The time-dependent electron energy density is calculated as:

$$
U_{\mathrm{CB}}(t)=\sum_{j>1}^{N} N_{0}\left(E_{j}-E_{1}\right) \rho_{j, j}(t),
$$

where $E_{1}$ is the energy associated to the lowest level of the conduction band (which associated kinetic energy is zero). Note that $U_{\mathrm{CB}}$ is evaluated by only accounting for transitions in the conduction band $(j>1)$, i.e., the absorbed energy during the photoionization process is not considered.

For all configurations, the laser electric field is chosen to be

$$
F(t)=\mathcal{F}_{0} \sin ^{2}\left(\pi t / \tau_{0}\right) \sin \left(\omega_{0} t\right),
$$

where $\omega_{0}, \tau_{0}$, and $\mathcal{F}_{0}$ are the laser frequency, the pulse duration, and the amplitude, respectively. The laser intensity is defined as $I_{0}=\varepsilon_{0} c \mathcal{F}_{0}^{2} / 2$, where $c$ is the speed of light and $\varepsilon_{0}$ is the vacuum permittivity. We consider a laser pulse with $\tau_{0}=70 \mathrm{fs}$, a photon energy $\hbar \omega_{0}$ varying from 0.6 to $3 \mathrm{eV}$, and peak intensities $I_{0}$ going from $1 \mathrm{TW} / \mathrm{cm}^{2}$ up to $500 \mathrm{TW} / \mathrm{cm}^{2}$.

\section{A. Photoionization}

The aim of this section is to evaluate the produced free electron density only induced by the photoionization process. We thus consider only two or three levels (one conduction level) to account for centrosymmetric or noncentrosymmetric materials. We emphasize that relaxation and impact ionization processes are not included here. Figure 1 presents the ionization degree as a function of the laser intensity for centrosymmetric and noncentrosymmetric materials and for several photon energies. The multiphoton absorption behavior (i.e., $Z \propto I_{0}^{K}$, where $K$ is the number of absorbed photons) is retrieved for $\hbar \omega_{0}=3,1.5,1 \mathrm{eV}$ for not too high intensities

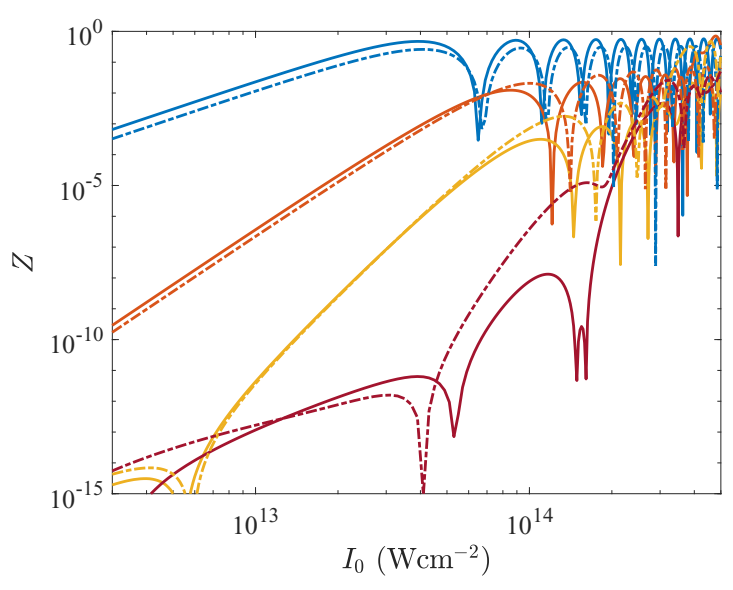

FIG. 1. Ionization degree as a function of the laser intensity for noncentrosymmetric material (solid curves) and centrosymmetric material (dashed curves) for several photon energies: $\hbar \omega_{0}=3.0 \mathrm{eV}$ (blue), $1.5 \mathrm{eV}$ (red), $1.0 \mathrm{eV}$ (orange), and $0.6 \mathrm{eV}$ (violet).

[a power-law fit provides $K=3,6,9$, respectively, which corresponds to the energy conservation $\left.K=E_{g} /\left(\hbar \omega_{0}\right)\right]$. For higher intensities, the perturbative conditions do not longer stand, leading to a departure of the power-law behavior as observed in Refs. [26,44]. For $\hbar \omega_{0}=0.6 \mathrm{eV}$, the behavior of $Z$ is different, where in particular a power law with a large exponent is not observed. In that case, for low intensities, the ionization process is driven by the high frequencies of the laser spectrum (not the central frequency $\omega_{0}$ ) which leads to a smaller multiphoton order [50]. The intensity increasing, the nonperturbative regime is quickly entered without a clear signature of the standard multiphoton absorption. Note that by taking another value of the transition matrix element only results in different absolute values of the produced electron density, while the overall behavior with respect to the intensity remains the same.

These results have also be compared to predictions of the Keldysh model (results not shown). The main discrepancy between both approaches is related to the wavelength dependence. For a given laser intensity, the OBE model predicts larger variations with the photon energy than the Keldysh theory. This is due to the description of the band structure. By including a dispersion relation for the bands, the Keldysh theory allows transitions out of the center of the Brillouin zone with various possible multiphoton order [15,51]. Thus changing the photon energy does not fully modify the quantum ionization paths. In case of OBE with flat bands, only one multiphoton order is involved. A change in the photon energy thus modifies the multiphoton order, and thus changes significantly the ionization rate. It is worth noting that for application purpose, the value of the dipolar matrix element can be adapted to account for any transition contributions out of the center of the Brillouin zone.

\section{B. Nonlinear polarization response}

This section is devoted to the polarization response of the material. Despite it is well-known that the OBE is able to account for this physical quantity [1,52-55], the present goal is to shed light on the ability of the present OBE model to 
account for the electron dynamics where specific material properties can be introduced, including material symmetry.

For that purpose, two examples are considered. For the first case, two VB levels $(j=-1,0)$ and one CB level $(j=1)$ with well-defined alternating parities as an example of centrosymmetric material. The dipole transition matrix $\hat{\mu}$ then is as follows:

$$
\left[\begin{array}{ccc}
0 & \mu_{-1,0} & 0 \\
\mu_{0,-1} & 0 & \mu_{0,1} \\
0 & \mu_{1,0} & 0
\end{array}\right] .
$$

The transition between the VB level $(j=-1)$ and the CB level is forbidden due to the same parity of these levels, i.e., the corresponding transition matrix element is zero. The allowed transitions between each pair of levels with the well-defined opposite parities is expected to only induce odd number of photons due to momentum conservation rule. For the second case, we consider one VB level $(j=0)$ and one CB level $(j=1)$. The VB level is chosen not to have a welldefined parity as an example of noncentrosymmetric material. The dipole transition matrix $\hat{\mu}$ then is as follows:

$$
\left[\begin{array}{cc}
\mu_{0,0} & \mu_{0,1} \\
\mu_{1,0} & 0
\end{array}\right] \text {. }
$$

It follows that both odd and even polarization responses are expected.

The time-dependent polarization response is obtained by calculating:

$$
P(t)=N_{0} \operatorname{Tr}\{e \hat{\mu}[\hat{\rho}-\hat{\rho}(0)]\},
$$

where $N_{0}$ is the density of neutrals. The initial condition is $P(t<0)=0$, which ensures the causality of the model [56,57]. In order to highlight the nonlinear optical response, a normalized polarization power spectrum is defined as:

$$
S(\omega)=\frac{|\mathcal{F}\{P(t)\}(\omega)|^{2}}{\left|\mathcal{F}\{P(t)\}\left(\omega_{0}\right)\right|^{2}},
$$

where $\mathcal{F}\{P(t)\}(\omega)$ denotes Fourier transforms. Figure 2 shows $S(\omega)$ induced by a 70 -fs laser pulse at $\hbar \omega_{0}=1.5 \mathrm{eV}$, and with various electric field amplitudes. The top and bottom graphs correspond to the previous three-level and two-level systems, respectively. For the three-level system, as expected, the polarization spectrum consisting of only odd harmonics due to the system symmetry, whereas both odd and even harmonics are generated with the two-level system. Figure 2 shows that the harmonics cut-off has a linear dependence on the incident electric field amplitude, which agrees with other works where the OBE were used for HHG simulations [54] and experiments [58]. Particularly, as in Ref. [58], the harmonics spectrum has well-pronounced maximum at the band-gap energy $E_{g}$. In general, the structure of the level system affects the HHG cut-off, i.e., in case of multilevel systems, several cut-offs in the HHG spectrum can be observed $[54,59,60]$. The present results also exhibit such a behavior.

\section{Full electron dynamics and energy deposition}

Here the full OBE model presented in Sec. II is used, including both photo- and impact ionization, electron heating, and recombination. The material modeling is done as
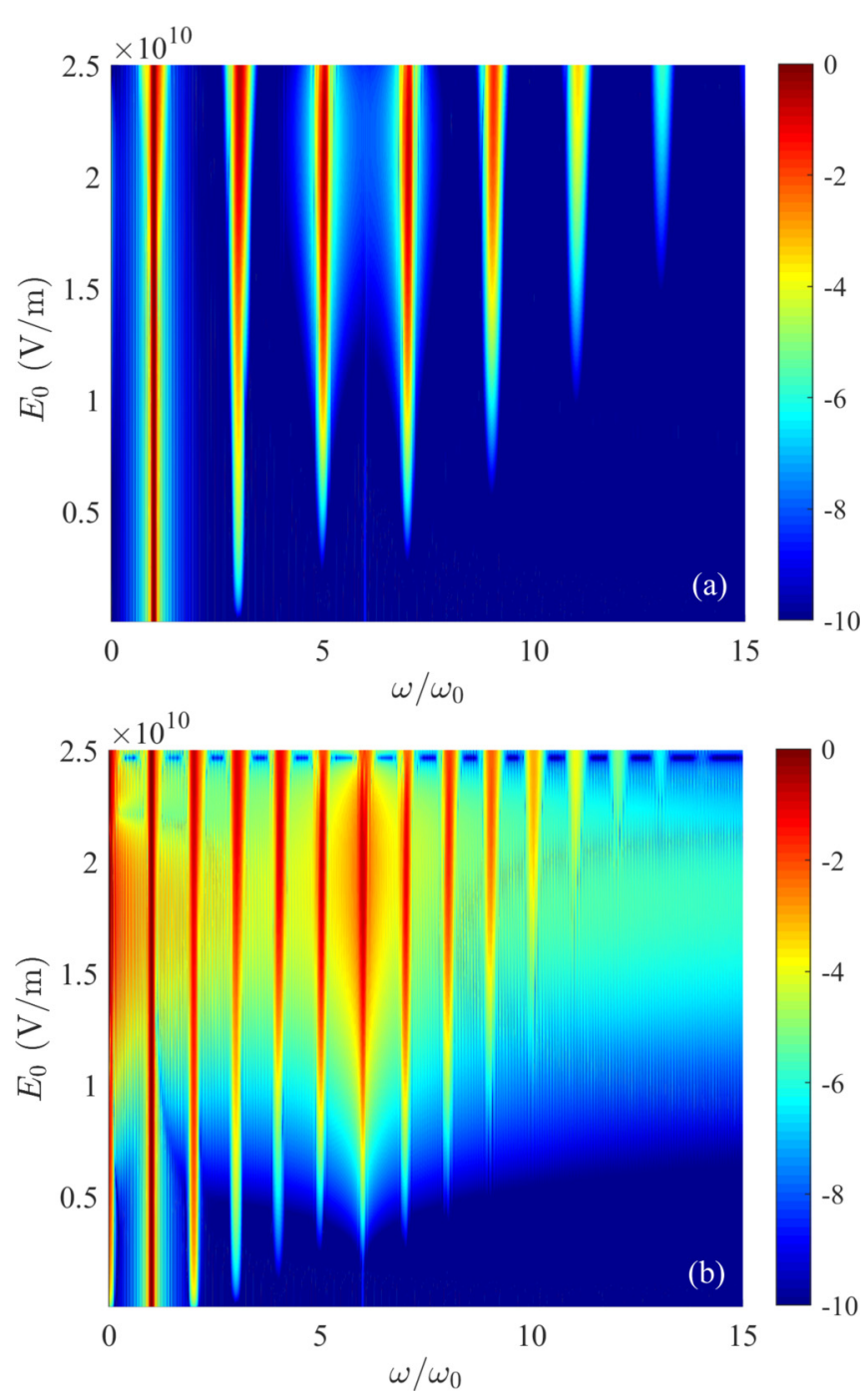

FIG. 2. Polarization spectrum induced by a 70 -fs laser pulse at $\hbar \omega_{0}=1.5 \mathrm{eV}$, and with various electric field amplitudes. The plotted quantity is $\log \left(|S(\omega)|^{2} /\left|S\left(\omega_{0}\right)\right|^{2}\right)$. (a) Material is described by a three-level system and by (b) two-level system (see text for details).

described in Sec. III, taking as many levels in the conduction band as necessary to allow impact ionization depending on the photon energy [see Eq. (7)]. A noncentrosymmetric material is considered here for illustration purpose, and the photon energies are $\hbar \omega=3,1.5,0.6 \mathrm{eV}$.

Figure 3 shows the temporal evolution of both electron density and energy density for the three photon energies. In each case, three calculations have been performed to exhibit the influence of various processes: Only the Liouvillevon Neumann operator is included (dotted blue curves), the same description with the coherence-loss process in addition (dashed red curves), and the full electron dynamics including all processes (solid black curves). In perspectives of applications of the present work to material modifications, the laser intensity has been set to obtain a deposited energy density of roughly $2 \mathrm{~kJ} / \mathrm{cm}^{3}$ at the end of the interaction (when full model is used), so that the intensity changes depending on the photon energy. Such an energy density corresponds to laser-induced damage threshold [18]. This criterion 

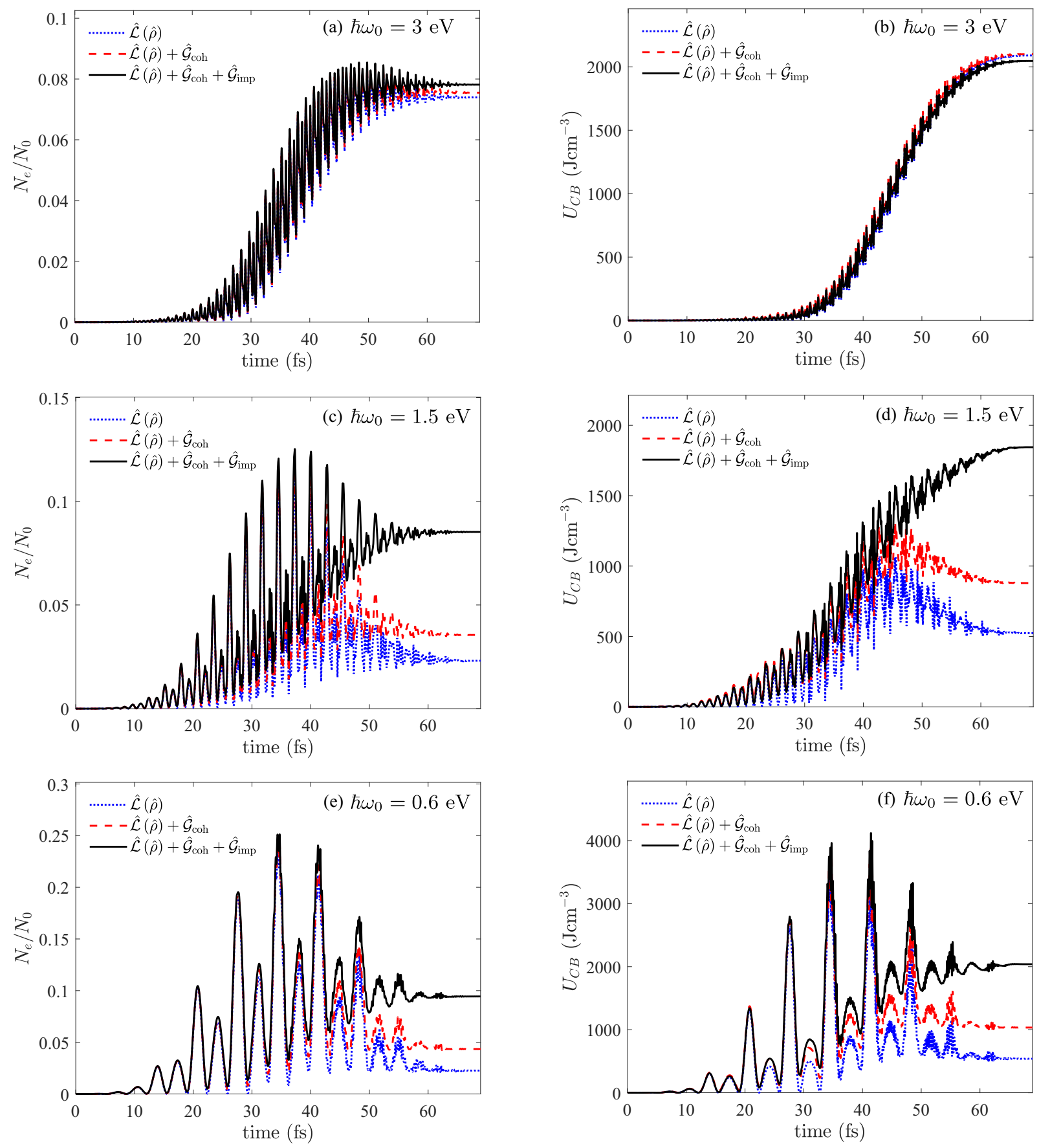

FIG. 3. Temporal evolution of [(a), (c), and (e)] the ionization degree $N_{e} / N_{0}$ and [(b), (d), and (f)] the energy gained by CB electrons $\mathrm{U}_{\mathrm{CB}}$ as predicted by the OBE model. Laser parameters are [(a) and (b) $] \hbar \omega_{0}=3 \mathrm{eV}$ and $I_{0}=2.0 \times 10^{13} \mathrm{~W} / \mathrm{cm}^{2},[(\mathrm{c})$ and $(\mathrm{d})] \hbar \omega_{0}=1.5 \mathrm{eV}$ and $I_{0}=1.3 \times 10^{14} \mathrm{~W} / \mathrm{cm}^{2}$, and $[(\mathrm{e})$ and (f) $] \hbar \omega_{0}=0.6 \mathrm{eV}$ and $I_{0}=2.15 \times 10^{14} \mathrm{~W} / \mathrm{cm}^{2}$. The intensity evolves with the photon energy to reach $2 \mathrm{~kJ} / \mathrm{cm}^{3}$ at the end of the pulse, corresponding to a threshold for material modifications (see text).

results in laser intensities of $2 \times 10^{13} \mathrm{~W} / \mathrm{cm}^{2}, 1.3 \times 10^{14}$ $\mathrm{W} / \mathrm{cm}^{2}$, and $2.15 \times 10^{14} \mathrm{~W} / \mathrm{cm}^{2}$ for $\hbar \omega=3,1.5,0.6 \mathrm{eV}$, respectively.

The evolutions of the electron density are first analysed. Regarding the case $\hbar \omega=3 \mathrm{eV}$ [see Fig. 3(a)], whatever the modeling configuration, the three curves exhibit the same shape: it consists of a monotonic increase in average as a function of time which mimics the intensity profile. Early and late in the pulse, the density variations are small, variations being the largest when the laser intensity is the highest, i.e., for times around 35 fs. This behavior corresponds to a standard evolution driven by photoionization [26]. In addition to this averaged behavior, oscillations of the density take place with a period of time corresponding to the period of an optical cycle. That corresponds to reversible ionization where the electron goes back and forth between valence and conduction bands as driven by the laser electric field, accounting for a classical electron dynamics [26]. The influence of loss 
of coherence and impact ionization should be to increase the electron density but it remains negligible. Despite electron heating takes place (because eventually $2 \mathrm{~kJ} / \mathrm{cm}^{3}$ are absorbed), this is the signature that electrons do not reach high-enough energy states to induce impact ionization. In the case $\hbar \omega_{0}=1.5 \mathrm{eV}$ [see Fig. 3(c)], each process contributes to the ionization. In the case where only the Liouville-von Neumann operator is included, the same behavior as the $\hbar \omega_{0}=3 \mathrm{eV}$ case is retrieved with only a higher amplitude for back-and-forth oscillations. That increase in this amplitude is consistent with the laser-induced classical dynamics of electron for which velocity scales as $\omega_{0}^{-1}$. Despite an intensity one order of magnitude larger than in the $\hbar \omega_{0}=3 \mathrm{eV}$ case, the final density is around 4 times less due to the largest multiphoton order. When the coherence loss mechanism is switched on, the overall density is increased. When valence electrons are transferred into the conduction band, they may undergo collisions with phonons resulting in breaking their coherence with the laser electric field so that they no longer come back to the valence band. When the possibility of impact ionization is included in the model, the overall density is further increased. In that case a large-enough electron density reaches energy levels allowing this process. Going to $\hbar \omega_{0}=0.6 \mathrm{eV}$ [see Fig. 3(e)], the overall behavior is similar to the case $\hbar \omega_{0}=1.5 \mathrm{eV}$. The only major difference is the increased period of oscillations which is consistent with the decrease in the photon energy. These results demonstrate that the present OBE model is able to describe the impact ionization process, and provides the expected dependence with respect to the laser wavelength: The longer the wavelength, the more probable the impact ionization. This behavior is consistent with standard approach as the Drude description where the electron heating is the most efficient for long wavelengths.

Now the evolution of the energy density is addressed [see Figs. 3(b), 3(d), and 3(f)]. Whatever the considered photon energy, the overall behavior of the energy density mimics the evolution of the electron density as expected. However, the influence of the impact ionization is different depending on the photon energy. For $\hbar \omega_{0}=3 \mathrm{eV}$ [see Fig. 3(b)] where the impact ionization process is not efficient, resulting in a negligible increase in the electron density compared to the pure photoionization configuration, its influence is to decrease the energy density. Each impact ionization event results from a single high-energy electron to two low-energy electrons. In addition within the present conditions, the laser heating is not sufficiently efficient to subsequently increase the overall energy. For $\hbar \omega_{0}=1.5 \mathrm{eV}$ [see Fig. 3(d)] and $\hbar \omega_{0}=0.6 \mathrm{eV}$ [see Fig. 3(f)], the impact ionization process results in an increase of the energy density compared to the model only including the photoionization process. In these configurations the loss in energy due to the impact ionization is compensated by a more efficient laser heating.

As a final comment regarding the temporal evolution of the electron energy density, we observe it also exhibits oscillations (mimicking those of the electron density). This behavior is due to the fact that a time-dependent approach is used, which departs from more standard modeling as the Drude approach or the one of Rethfeld [16] which predict a monotonic increase in the energy density with respect to time.
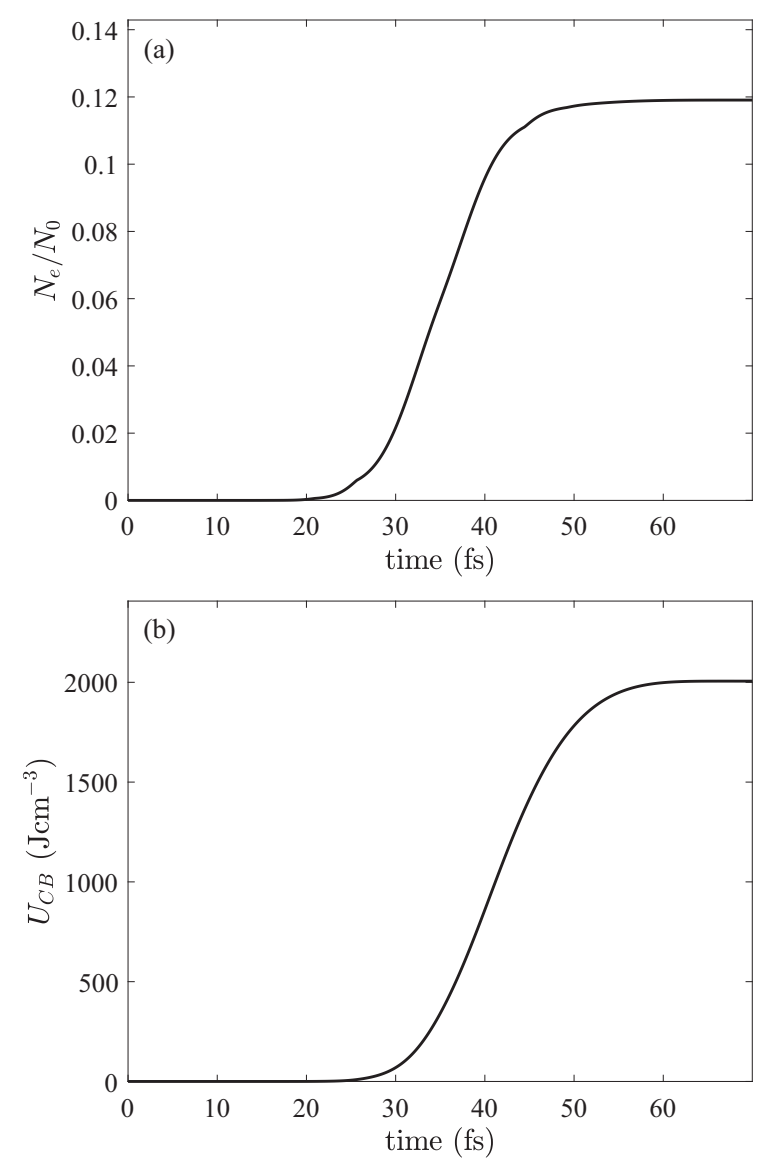

FIG. 4. Temporal evolution of (a) the ionization degree $N_{e} / N_{0}$ and (b) the energy gained by $\mathrm{CB}$ electrons $\mathrm{U}_{\mathrm{CB}}$ as predicted by a model based on multiple rate equations [16]. The laser parameters are $\hbar \omega_{0}=1.5 \mathrm{eV}$ and $I_{0}=1.3 \times 10^{14} \mathrm{~W} / \mathrm{cm}^{2}$. The intensity has been chosen such that $U_{\mathrm{CB}} \simeq 2 \mathrm{~kJ} / \mathrm{cm}^{3}$ at the end of the pulse.

This trend is due to the fact that these classes of approach rely on a temporal average over a few optical cycles for every process including photoionization [15] and laser-induced electron dynamics in the conduction band [16]. Figure 4 shows both electron density and energy density for $\hbar \omega_{0}=$ $1.5 \mathrm{eV}$ and $I_{0}=1.3 \times 10^{14} \mathrm{~W} / \mathrm{cm}^{2}$, as predicted by Rethfeld model [16], which indeed evolve monotonically with respect to time. Within this approach the photoionization is described by the Keldysh expression, and the electron dynamics in the conduction band is described by a set of rate equations coupled by a one-photon absorption process which amplitude is provided by a constant cross section. Note that in this case of $70 \mathrm{fs}$ pulse with a relatively large number of optical cycles, the average over a few optical cycles of the curves predicted by the full OBE model allows us to retrieve the behavior predicted by the Rethfeld approach, which further validate the present OBE model (similar trends are obtained for the other photon energies). In case of few-cycle pulses, as expected the comparison between results of OBE and multiple rate equations no longer stands (results not shown) because the assumption of averaging over optical cycles breaks. The description of the electron dynamics in such an extreme condition thus requires a time-dependent model as the proposed OBE one. 


\section{CONCLUSION}

In order to describe the time-dependent electron dynamics in dielectric materials induced by femtosecond laser pulses, a model based on optical Bloch equations has been developed. Through the introduction of an appropriate set of energy levels mimicking the band structure of dielectric materials, the present approach includes a description of photoionization, electron heating in the conduction band, impact ionization, collisions in the conduction band, and electron recombination. The reliability of this approach has been assessed by studying various physical quantities. First, the evolution of the density of the conduction electrons with respect to the laser intensity in case of pure photoionization has been studied. By changing the wavelength, we have shown that this model is able to account for the multiphoton absorption in the perturbative case, i.e., not too high intensities. For intensities in excess of $10^{14} \mathrm{~W} / \mathrm{cm}^{2}$, the tunneling regime is entered leading to a saturation of the ionization rate with respect to the intensity. Second, the evaluation of the polarization has been provided. Its Fourier transform confirms that harmonic generation is well described up to high orders. In particular, it is demonstrated that properties of symmetry of the material lattice can be included: Only odd harmonics are generated by imposing a centrosymmetric structure. Finally, the full electron dynamics has been studied. The temporal evolution of the density of conduction electrons clearly exhibits the ability of this modeling to describe the time-dependent electron dynamics driven by the oscillating electric laser field. The electron energy density is shown to follow such a behavior. The influence of the impact ionization is also retrieved, demonstrating the ability of the present model to account for this key process. As expected, the lower the photon energy, the larger its contribution.

Overall, the present modeling describes all expected behaviors regarding the laser-induced electron dynamics in case where all main collisional processes are included. This approach thus provides a theoretical baseline well adapted to describe accurately the electron dynamics driven by femtosecond laser pulse. Such an approach is well adapted to be introduced in a Maxwell solver to describe the coupled electron-pulse propagation dynamics (see Appendix). Such a coupling will allow one to provide accurate predictions of the energy deposition in dielectric materials. It may thus provide a step forward for numerically designing experiments and applications.

\section{ACKNOWLEDGMENTS}

The French Region Nouvelle-Aquitaine (MOTIF project) and the DGA (AS-TGV project) are acknowledged for supporting this work. Numerical simulations were performed using computing resources at Grand Équipement National pour le Calcul Intensif (GENCI, Grants No. A0030506129 and No. A0040507594) and Chalmers Centre for Computational Science and Engineering (C3SE) provided by the Swedish National Infrastructure for Computing (SNIC, Grant No. SNIC 2018/4-38). This work was supported by Russian Science Foundation (RSF) (Projects No. 17-72-20130).

\section{APPENDIX: NUMERICAL RESOLUTION OF OBE}

In the context of finite-difference time-domain (FDTD) Maxwell-Bloch simulations, the OBE are coupled to a Maxwell solver, e.g., the Yee scheme [61]. In this section, we present the numerical scheme used in this paper to solve OBE equations and which can be easily integrated into such FDTD algorithms.

Let us consider that the electric field $E(t)$ is known, in general from the Maxwell solver and particularly in this paper from Eq. (17), at the discrete instants $\left\{t_{n}=n \Delta t\right\}$, where $\Delta t$ is the time step and $n \geqslant 0$. This instants will constitute the primal temporal grid. At these primal instants the Hamiltonian is fully known according to Eqs. (3) and (8):

$$
\hat{H}\left(t_{n}\right)=\hat{H}_{0}-e F\left(t_{n}\right) \hat{\mu} .
$$

FDTD codes usually update alternatively the electric field $F(t)$ and material response variables [i.e., the electron density $N_{e}(t)$ and the current density $\left.J(t)\right]$. In consequence, we need to construct a numerical scheme allowing us to compute $\hat{\rho}$ at the discrete instants $\left\{t_{n-1 / 2}=t_{n}-\Delta t / 2\right\}$, which will constitute the dual temporal grid. All the material response variables can be computed from the density matrix. We shall use the notation $\hat{\rho}_{n-1 / 2}$ for the numerical computation of the density matrix at dual instant $t_{n-1 / 2}$. For centrosymmetric material, we impose as initial condition that all the two VB levels are equally populated and that all the $N$ CB levels are completely depleted:

$$
\hat{\rho}_{-1 / 2}=\left[\begin{array}{ccccc}
\frac{1}{2} & 0 & 0 & 0 & 0 \\
0 & \frac{1}{2} & 0 & 0 & 0 \\
0 & 0 & 0 & 0 & 0 \\
0 & 0 & 0 & \ddots & 0 \\
0 & 0 & 0 & 0 & 0
\end{array}\right] .
$$

During each update step, in order to calculate $\hat{\rho}_{n+1 / 2}$ from $\hat{\rho}_{n-1 / 2}$ and $\hat{H}\left(t_{n}\right)$, each of the operators in Eq. (1) is treated separately following the Strang splitting approach [62]. We seek a second-order accurate scheme at every iteration and, since the numerical cost of applying the relaxation operators is considerably smaller than applying the Liouville-von Neumman operator, we update the density matrix in seven substeps as follows:

$$
\begin{aligned}
& \hat{\rho}_{n+1 / 2}^{(1)}=\hat{G}_{\mathrm{rec}}^{\Delta t / 2}\left(\hat{\rho}_{n-1 / 2}\right), \\
& \hat{\rho}_{n+1 / 2}^{(2)}=\hat{G}_{\mathrm{imp}}^{\Delta t / 2}\left(\hat{\rho}_{n+1 / 2}^{(1)}\right), \\
& \hat{\rho}_{n+1 / 2}^{(3)}=\hat{G}_{\mathrm{coh}}^{\Delta t / 2}\left(\hat{\rho}_{n+1 / 2}^{(2)}\right), \\
& \hat{\rho}_{n+1 / 2}^{(4)}=\hat{L}^{\Delta t}\left(\hat{\rho}_{n+1 / 2}^{(3)}, \hat{H}\left(t_{n}\right)\right), \\
& \hat{\rho}_{n+1 / 2}^{(5)}=\hat{G}_{\mathrm{coh}}^{\Delta t / 2}\left[\hat{\rho}_{n+1 / 2}^{(4)}\right], \\
& \hat{\rho}_{n+1 / 2}^{(6)}=\hat{G}_{\mathrm{imp}}^{\Delta t / 2}\left[\hat{\rho}_{n+1 / 2}^{(5)}\right], \\
& \hat{\rho}_{n+1 / 2}=\hat{G}_{\mathrm{rec}}^{\Delta t / 2}\left[\hat{\rho}_{n+1 / 2}^{(6)}\right],
\end{aligned}
$$

where $\hat{G}_{\text {rec }}^{\Delta t}$ is the discrete recombination operator acting over $\Delta t, \hat{G}_{\mathrm{imp}}^{\Delta t}$ is the discrete impact-ionization operator acting over $\Delta t, \hat{G}_{\text {coh }}^{\Delta t}$ is the discrete coherence-loss operator acting over $\Delta t$, and $L^{\Delta t}$ is the discrete Liouville-von 
Neumann operator acting over $\Delta t$. In order to preserve the properties of the density matrix over the simulation time span, the discretization of the ensemble of operators during each update step must constitute a completely positive trace preserving (CPTP) map [63,64]. In order to have a CPTP splitting in Eq. (A3) and thus obtain numerical solutions compatible with physics, we must assure that all the discrete operators (namely, $\hat{G}_{\text {rec }}^{\Delta t}, \hat{G}_{\text {imp }}^{\Delta t}, \hat{G}_{\text {coh }}^{\Delta t}$, and $\hat{L}^{\Delta t}$ ) are CPTP [65].

In case of two VB levels, the recombination operator introduces recombination process from each $\mathrm{CB}$ level to each VB level $(k=0, k=-1)$ with the same characteristic time scale $\tau_{\text {rec }}$ similarly to the case of one VB [Eq. (10)] and is discretized as follows:

$$
\hat{G}_{\mathrm{rec}}^{\Delta t}(\hat{\rho})=\sum_{k=-1}^{0} \hat{G}_{\mathrm{rec} \mathrm{k}}^{\Delta t}(\hat{\rho}),
$$

where

$$
\hat{G}_{\text {rec k }}^{\Delta t}(\hat{\rho}) \equiv \begin{cases}\rho_{j, j}=\rho_{j, j}-\Delta \rho_{j, j}, & \text { for } j>0 \\ \rho_{k, k}=\rho_{k, k}+\sum_{j=1}^{N} \Delta \rho_{j, j}, & \end{cases}
$$

where $\Delta \rho_{j, j}=\left(1-e^{-\Delta t / \tau_{\text {rec }}}\right) \rho_{j, j}$ is the decay in the electron population in the $j$ th $\mathrm{CB}$ level over the time $\Delta t$ due to recombination transition to the $k$ th $\mathrm{VB}$ level. It is straightforward to verify that this discretization of the recombination operator conserves the trace and the positivity of the diagonal elements of $\hat{\rho}$.

Following Eq. (12), the impact-ionization operator acting over a time $\Delta t$ on a system with two VB levels is discretized as follows:

$$
\hat{G}_{\text {imp }}^{\Delta t}(\hat{\rho}) \equiv\left\{\begin{array}{l}
\rho_{N, N}=\rho_{N, N}-\sum_{k=-1}^{0} \Delta \rho_{k, k}, \\
\rho_{1,1}=\rho_{1,1}+\sum_{k=-1}^{0} 2 \Delta \rho_{k, k}, \\
\rho_{k, k}=\rho_{k, k}-\Delta \rho_{k, k},
\end{array} \quad \text { for } k \leqslant 0,\right.
$$

where, here, $\Delta \rho_{k, k}$ represents the decay in the electron population of the $k$ th VB level and is given by:

$$
\Delta \rho_{k, k}=\min \left\{\rho_{k, k},\left(1-e^{-\Delta t / \tau_{\text {imp }}}\right) \rho_{N, N}\right\} .
$$

The introduction of the min-function in Eq. (A7) when computing $\Delta \rho_{k, k}$ ensures the preservation of the nonnegativeness of all VB levels: $\rho_{k, k} \geqslant 0$ for $-1 \leqslant k \leqslant 0$.

The discretization of the coherence-loss operator, given by Eq. (11), acting over a time $\Delta t$, is the following:

$$
\hat{G}_{\mathrm{coh}}^{\Delta t}(\hat{\rho}) \equiv\left\{\rho_{j, k}=e^{-\Delta t / \tau_{\mathrm{coh}}} \rho_{j, k}, \quad \text { for } j \neq k,\right.
$$

which preserves all the properties of density matrix $\hat{\rho}$.

There are several possibilities of obtaining a CPTP discrete Liouville-von Neumann operator, such as the Crank-Nicolson approach [66,67], Runge-Kutta methods [68-70], and the matrix exponential approach $[26,65]$. The latter technique is chosen in this paper because it adapts well to the alternatively updating nature of FDTD codes. Assuming that during the time interval $\left[t_{n-1 / 2}, t_{n+1 / 2}\right]$ the Hamiltonian in the Liouvillevon Neumann operator is time-independent and equal to $\hat{H}\left(t_{n}\right)$ given by Eq. (A1), then the exact solution to $\partial_{t} \hat{\rho}=\hat{\mathcal{L}}(\hat{\rho})$ reads:

$$
\hat{\rho}(t)=\hat{\mathcal{H}}(t) \hat{\rho}_{n-1 / 2} \hat{\mathcal{H}}(t)^{\dagger},
$$

where the $\hat{\mathcal{H}}(t)$ is the following matrix exponential:

$$
\hat{\mathcal{H}}(t)=\exp \left[-\frac{i}{\hbar} \hat{H}\left(t_{n}\right)\left(t-t_{n-1 / 2}\right)\right] .
$$

Since $H\left(t_{n}\right)$ is real and symmetric in our paper, $\hat{\mathcal{H}}(t)^{\dagger}$ reduces to $\hat{\mathcal{H}}(t)^{*}$ in Eq. (A9). Therefore, discretization of the Liouville-von Neumann operator in Eq. (A3) reads as follows:

$$
\hat{L}^{\Delta t}(\hat{\rho}, \hat{H}) \equiv\left\{\hat{\rho}=\hat{\mathcal{H}} \hat{\rho} \hat{\mathcal{H}}^{*},\right.
$$

where

$$
\hat{\mathcal{H}}=\hat{\mathcal{H}}(\Delta t, \hat{H})=\exp \left[-\frac{i}{\hbar} \hat{H} \Delta t\right]
$$

which is CPTP because it is an exact solution of the Liouvillevon Neumann equation. In practice, since the numerical calculation of matrix exponentials requires high computational ressources [71], we can use a second-order accuratein-time approximation of Eq. (A12) provided that the norm $\|-i \hat{H} \Delta t / \hbar\| \ll 1$ for a time step $\Delta t$ being sufficiently small. The approximation that we use in this paper, which is CPTP, reads as follows [26]:

$$
\exp \left[-\frac{i}{\hbar} \hat{H} \Delta t\right] \approx \hat{\mathcal{I}}\left(\hat{\mathcal{I}}^{*}\right)^{-1}+\mathcal{O} \Delta t^{3}
$$

where

$$
\hat{\mathcal{I}}=\hat{I}-\frac{i \Delta t}{2 \hbar} \hat{H}
$$

and $\hat{I}$ is the identity matrix. Thanks to the approximation (A13) only one matrix inversion and two matrix conjugations are computed at each time iteration.

Finally, we compute the material response variables from the density matrix at the dual instants. The electron density can be easily calculated thanks to Eq. (15):

$$
N_{e}\left(t_{n+1 / 2}\right)=N_{0} \sum_{j>0}^{N} \rho_{j, j}\left(t_{n+1 / 2}\right) .
$$

And the polarization response is calculated accordingly to Eq. (18):

$$
P\left(t_{n+1 / 2}\right)=N_{0} \operatorname{Tr}\left\{e \hat{\mu}\left[\hat{\rho}\left(t_{n+1 / 2}\right)-\hat{\rho}(0)\right]\right\}
$$

The current density is then computed as a time derivative of the polarization response :

$$
\begin{aligned}
J\left(t_{n+1 / 2}\right)= & N_{0} \operatorname{Tr}\left\{-\frac{i}{\hbar} e \hat{\mu}\left[\frac{\hat{H}\left(t_{n}\right)+\hat{H}\left(t_{n+1}\right)}{2} \hat{\rho}_{n+1 / 2}\right]\right. \\
& +e \hat{\mu} \hat{\mathcal{G}}_{\mathrm{rec}}\left(\hat{\rho}_{n+1 / 2}\right)+e \hat{\mu} \hat{\mathcal{G}}_{\mathrm{coh}}\left(\hat{\rho}_{n+1 / 2}\right) \\
& \left.+e \hat{\mu} \hat{\mathcal{G}}_{\mathrm{imp}}\left(\hat{\rho}_{n+1 / 2}\right)\right\}
\end{aligned}
$$


[1] G. Ndabashimiye, S. Ghimire, M. Wu, D. A. Browne, K. J. Schafer, M. B. Gaarde, and D. A. Reis, Nature 534, 521 (2016).

[2] G. Vampa, C. R. McDonald, G. Orlando, D. D. Klug, P. B. Corkum, and T. Brabec, Phys. Rev. Lett. 113, 073901 (2014).

[3] Y. S. You, Y. C. Yin, Y. Wu, A. Chew, X. M. Ren, F. J. Zhuang, S. Gholam-Mirzaei, M. Chini, Z. H. Chang, and S. Ghimire, Nat. Commun. 8, 724 (2017).

[4] M. Hohenleutner, F. Langer, O. Schubert, M. Knorr, U. Huttner, S. W. Koch, M. Kira, and R. Huber, Nature 523, 572 (2015).

[5] O. Schubert, M. Hohenleutner, F. Langer, B. Urbanek, C. Lange, U. Huttner, D. Golde, T. Meier, M. Kira, S. W. Koch, and R. Huber, Nat. Photon. 8, 119 (2014).

[6] B. Chimier, O. Utéza, N. Sanner, M. Sentis, T. Itina, P. Lassonde, F. Légaré, F. Vidal, and J. C. Kieffer, Phys. Rev. B 84, 094104 (2011).

[7] A. Rudenko, J.-P. Colombier, S. Hohm, A. Rosenfeld, J. Kruger, J. Bonse, and T. E. Itina, Sci. Rep. 7, 12306 (2017).

[8] K. J. Wædegaard, D. B. Sandkamm, A. Mouskeftaras, S. Guizard, and P. Balling, Europhys. Lett. 105, 47001 (2014).

[9] M. Schultze, E. M. Bothschafter, A. Sommer, S. Holzner, W. Schweinberger, M. Fiess, M. Hofstetter, R. Kienberger, V. Apalkov, V. Yakovlev, M. I. Stockman, and F. Krausz, Nature 493, 75 (2013).

[10] J. R. Gulley and T. E. Lanier, Phys. Rev. B 90, 155119 (2014).

[11] A. Patel, Y. Svirko, C. Durfee, and P. G. Kazansky, Sci. Rep. 7, 12928 (2017).

[12] A. S. Arabanian and R. Massudi, J. Opt. Soc. Am. B 31, 748 (2014).

[13] D. N. Vitek, E. Block, Y. Bellouard, D. E. Adams, S. Backus, D. Kleinfeld, C. G. Durfee, and J. A. Squier, Opt. Express 18, 24673 (2010).

[14] P. A. Zhokhov and A. M. Zheltikov, Sci. Rep. 8, 1824 (2018).

[15] L. V. Keldysh, Zh. Eksp. Teor. Fiz. 47, 1945 (1964) [Sov. Phys. JETP 20, 1307 (1965)].

[16] B. Rethfeld, Phys. Rev. Lett. 92, 187401 (2004).

[17] B. H. Christensen and P. Balling, Phys. Rev. B 79, 155424 (2009).

[18] L. Gallais, D.-B. Douti, M. Commandré, G. Batavičiūte, E. Pupka, M. Ščiuka, L. Smalakys, V. Sirutkaitis, and A. Melninkaitis, J. Appl. Phys. 117, 223103 (2015).

[19] G. Duchateau and A. Bourgeade, Phys. Rev. A 89, 053837 (2014).

[20] N. Bulgakova, R. Stoian, A. Rosenfeld, I. Hertel, W. Marine, and E. Campbell, Appl. Phys. A 81, 345 (2005).

[21] N. M. Bulgakova, V. P. Zhukov, S. V. Sonina, and Y. P. Meshcheryakov, J. Appl. Phys. 118, 233108 (2015).

[22] I. Mirza, N. M. Bulgakova, J. Tomastik, V. Michalek, O. Haderka, L. Fekete, and T. Mocek, Sci. Rep. 6, 39133 (2016).

[23] B. Bidégaray, A. Bourgeade, and D. Reignier, J. Comput. Phys. 170, 603 (2001).

[24] M. Kolesik, J. V. Moloney, and M. Mlejnek, Phys. Rev. Lett. 89, 283902 (2002).

[25] M. Kolesik and J. V. Moloney, Phys. Rev. E 70, 036604 (2004).

[26] A. Bourgeade and G. Duchateau, Phys. Rev. E 85, 056403 (2012).

[27] J. R. Peñano, P. Sprangle, B. Hafizi, W. Manheimer, and A. Zigler, Phys. Rev. E 72, 036412 (2005).

[28] A. Bourgeade and O. Saut, J. Comput. Phys. 213, 823 (2006).
[29] J. Tolliver and M. Kolesik, Opt. Express 26, 30172 (2018).

[30] A. Kaiser, B. Rethfeld, M. Vicanek, and G. Simon, Phys. Rev. B 61, 11437 (2000).

[31] L. Barilleau, G. Duchateau, B. Chimier, G. Geoffroy, and V. Tikhonchuk, J. Phys. D: Appl. Phys. 49, 485103 (2016).

[32] M. Qasim, M. S. Wismer, M. Agarwal, and V. S. Yakovlev, Phys. Rev. B 98, 214304 (2018).

[33] S. A. Sato, K. Yabana, Y. Shinohara, T. Otobe, K.-M. Lee, and G. F. Bertsch, Phys. Rev. B 92, 205413 (2015).

[34] K.-M. Lee, C. Min Kim, S. A. Sato, T. Otobe, Y. Shinohara, K. Yabana, and T. Moon Jeong, J. Appl. Phys. 115, 053519 (2014).

[35] T. Kumada, H. Akagi, R. Itakura, T. Otobe, and A. Yokoyama, J. Appl. Phys. 115, 103504 (2014).

[36] M. Lamperti, V. Jukna, O. Jedrkiewicz, P. Di Trapani, R. Stoian, T. E. Itina, C. Xie, F. Courvoisier, and A. Couairon, APL Photon. 3, 120805 (2018).

[37] S. Tzortzakis, L. Sudrie, M. Franco, B. Prade, A. Mysyrowicz, A. Couairon, and L. Bergé, Phys. Rev. Lett. 87, 213902 (2001).

[38] M. V. Shugaev, C. Wu, O. Armbruster, A. Naghilou, N. Brouwer, D. S. Ivanov, T. J.-Y. Derrien, N. M. Bulgakova, W. Kautek, B. Rethfeld et al., MRS Bull. 41, 960 (2016).

[39] N. Bulgakova and V. Zhukov, Continuum Models of Ultrashort Laser-Matter Interaction in Application to Wide-Bandgap Dielectrics, Lasers in Materials Science, Springer Series in Materials Science ed., Vol. 191 (Springer, Cham, 2014).

[40] O. Dematteo Caulier, K. Mishchik, B. Chimier, S. Skupin, A. Bourgeade, C. Javaux Léger, R. Kling, C. Hönninger, J. Lopez, V. Tikhonchuk, and G. Duchateau, Appl. Phys. Lett. 107, 181110 (2015).

[41] R. Beuton, B. Chimier, J. Breil, D. Hébert, P.-H. Maire, and G. Duchateau, J. Appl. Phys. 122, 203104 (2017).

[42] E. Gamaly, Phys. Rep. 508, 91 (2011).

[43] R. W. Boyd, Nonlinear Optics (3rd. ed.) (Academic Press, San Diego, CA, 2008).

[44] S. A. Sato, M. Lucchini, M. Volkov, F. Schlaepfer, L. Gallmann, U. Keller, and A. Rubio, Phys. Rev. B 98, 035202 (2018).

[45] S. Y. Kruchinin, F. Krausz, and V. S. Yakovlev, Rev. Mod. Phys. 90, 021002 (2018).

[46] A. Q. Wu, I. H. Chowdhury, and X. F. Xu, Phys. Rev. B 72, 085128 (2005).

[47] P. Audebert, P. Daguzan, A. Dos Santos, J. C. Gauthier, J. P. Geindre, S. Guizard, G. Hamoniaux, K. Krastev, P. Martin, G. Petite, and A. Antonetti, Phys. Rev. Lett. 73, 1990 (1994).

[48] J. Rolle, L. Bergé, G. Duchateau, and S. Skupin, Phys. Rev. A 90, 023834 (2014).

[49] D. Arnold, E. Cartier, and D. J. DiMaria, Phys. Rev. B 45, 1477 (1992).

[50] H. Jouin and G. Duchateau, Phys. Rev. A 99, 013433 (2019).

[51] C. Mézel, G. Duchateau, G. Geneste, and B. Siberchicot, J. Phys.: Condens. Matter 25, 235501 (2013).

[52] P. G. Hawkins, M. Y. Ivanov, and V. S. Yakovlev, Phys. Rev. A 91, 013405 (2015).

[53] D. Golde, T. Meier, and S. W. Koch, Phys. Rev. B 77, 075330 (2008).

[54] M. Wu, S. Ghimire, D. A. Reis, K. J. Schafer, and M. B. Gaarde, Phys. Rev. A 91, 043839 (2015).

[55] J. B. Khurgin, J. Opt. Soc. Am. B 33, C1 (2016).

[56] C. W. Peterson and B. W. Knight, J. Opt. Soc. Am. 63, 1238 (1973).

[57] B. Y. Hu, Am. J. Phys. 57, 821 (1989). 
[58] E. N. Osika, A. Chacón, L. Ortmann, N. Suárez, J. A. Pérez-Hernández, B. Szafran, M. F. Ciappina, F. Sols, A. S. Landsman, and M. Lewenstein, Phys. Rev. X 7, 021017 (2017).

[59] C. R. McDonald, G. Vampa, P. B. Corkum, and T. Brabec, Phys. Rev. A 92, 033845 (2015).

[60] M. Wu, D. A. Browne, K. J. Schafer, and M. B. Gaarde, Phys. Rev. A 94, 063403 (2016).

[61] K. S. Yee, IEEE Trans. Antennas Propag. 14, 302 (1966).

[62] G. Strang, SIAM J. Numer. Anal. 5, 506 (1968).

[63] G. Lindblad, Commun. Math. Phys. 48, 119 (1976).

[64] V. Gorini, A. Kossakowski, and E. C. G. Sudarshan, J. Math. Phys. 17, 821 (1976).
[65] M. Riesch and C. Jirauschek, J. Comp. Phys. 390, 290 (2019).

[66] R. W. Ziolkowski, J. M. Arnold, and D. M. Gogny, Phys. Rev. A 52, 3082 (1995).

[67] G. Slavcheva, J. M. Arnold, I. Wallace, and R. W. Ziolkowski, Phys. Rev. A 66, 063418 (2002).

[68] M. Sukharev and A. Nitzan, Phys. Rev. A 84, 043802 (2011).

[69] A. Deinega and T. Seideman, Phys. Rev. A 89, 022501 (2014).

[70] W. Cartar, J. Mørk, and S. Hughes, Phys. Rev. A 96, 023859 (2017).

[71] C. Moler and C. V. Loan, SIAM Rev. 45, 3 (2003). 